\title{
The influence of air humidity on effectiveness of heat sink work
}

by M. Kopeć*, R. Olbrycht*

* Lodz Univ. of Technology, 90-924, 211/215 Wolczanska Str., Lodz, Poland, michal.kopec@dokt.p.lodz.pl, robert.olbrycht@p.lodz.pl

\begin{abstract}
The aim of this paper is to demonstrate the influence of the air humidity on the effectiveness of heat sink work. It was observed that higher air humidity enabled higher power dissipation in the transistor without increasing its junction temperature. The presented simulation results additionally show the dependence between air humidity and estimated value of heat flux. The test rig was elaborated at the Institute of Electronics (Lodz University of Technology). In addition, this rig enabled visualisation of natural convection in different infrared spectral ranges. Multispectral approach revealed that this convection air flow is visible in the most clear way in MWIR spectral range with cooled camera.
\end{abstract}

\section{Introduction}

The heat dissipation from a transistor is provided by a package and using the heat sink. The effectiveness of this process is dependent on many factors, e.g. the material from which the heat sink was made, its shape, color, active surface and also the contact quality between the package and the heat sink [1,2]. This contact is dependent on used insulator and thermal paste (grease). In practice the junction temperature value $T_{J}$ of the transistor should be below maximum value from the datasheet (typically $150{ }^{\circ} \mathrm{C}$ ). Hence there is often a need for heat sink with large surface and sometimes even forced cooling [3]. Sometimes use of the large heat sink is impossible, because size of the device is too small.

The total thermal resistance between the junction of the semiconductor element and the ambient is given by (1) and the temperature value of the junction is given by (2).

$$
\begin{gathered}
R_{t h j a}=R_{t h j c}+R_{t h c r}+R_{t h r a} \\
T_{j}=T_{a}+P\left(R_{t h j c}+R_{t h c r}+R_{t h r a}\right)
\end{gathered}
$$

where:

j - semiconductor junction,

c - case (package),

$r \quad$ - radiator (heat sink),

a - ambient,

P - power dissipated in the transistor.

Let us take an exemplary transistor in TO-218 package - typical values of the thermal resistance are: $R_{\text {thjc }}=1 \ldots 3 \mathrm{KNW}, R_{\text {thcr }}$ is typically about $1 \mathrm{KN}, R_{\text {thra }}$ is from $0,5 \mathrm{KM}$ for large heat sink to $50 \mathrm{~K} / \mathrm{W}$ for small heat sink. While $R_{\text {thjc }}$ value cannot be reduced by user as it is a technological limitation, the two other resistances may be decreased respectively by using a paste (grease) that fills microscopic irregularities on the surface of the heat sink and increasing its cooling effectiveness. This paper proposes a method for improving efficacy of the heat sink by increasing the air humidity. It may be done e.g. by producing a water vapour with using an ultrasonic generator.

\section{Simulations}

The modeled system consist of an aluminum heat sink for transistor cooling, where the contact is provided with a thermal grease (fig. 1). It is multi-physics model to solve the heat transfer from the semiconductor junction to the surrounding air or water - for different concentrations of water vapour, one needs to interpolate obtained results.

The temperature is solved through the heat equation and the heat transport is occurred through conduction and convection (3). The conduction is considered only in transistor and heat sink walls in order to maintain energy balance. The change in energy is equal to the heat source minus the divergence of the diffusive heat flux. The temperature $T_{0}$ as boundary condition was set at the junction of the transistor, equal to $150^{\circ} \mathrm{C}$ (this condition was also maintained during the real measurements). The air properties dependent on the time are the density, the thermal conductivity and the heat capacity. When calculating the temperature for the case of the stationary state, then the first term of the equations (3) disappears. 
where:

$$
p C_{p} \frac{\partial T}{\partial t}+p C_{p} u * \nabla T=\nabla *(\mathbf{k} \nabla \mathrm{T})+\mathbf{Q}
$$

$p-$ density, $\mathrm{kg} / \mathrm{m}^{3}$,

$C_{p}$ - specific heat capacity at constant pressure, $\mathrm{J} /\left(\mathrm{kg}^{*} \mathrm{~K}\right)$,

$T$ - absolute temperature, $\mathrm{K}$,

$t$ - time, s,

$u$ - velocity vector, $\mathrm{m} / \mathrm{s}$,

$Q$ - heat sources other than viscous heating, $\mathrm{W} / \mathrm{m}^{3}$.

The heat equation accepts two basic types of boundary conditions: the specified temperature (4) and the specified heat flux (5). Both boundary conditions were used in the model.

$$
\begin{gathered}
T=T_{0} \\
-n * q=q_{0}
\end{gathered}
$$

where:

$q-$ conductive heat flux vector $\left(\mathrm{W} / \mathrm{m}^{2}\right)$, where $\mathrm{q}$ is $(-\mathbf{k} \nabla \mathbf{T})$. The thermal conductivity describes the relationship between the heat flux vector $q$ and the temperature gradient $\nabla T$ in the form of $q=-k \nabla T$, which is Fourier's law of the heat conduction,

$n$ - the normal vector of the boundary,

$q_{0}$ - inward heat flux $\left(\mathrm{W} / \mathrm{m}^{2}\right)$, normal to the boundary.

The simulation included the effect of an exterior fluid (5). For water, there is taken into consideration a wall height $L$ and external temperature $T_{\text {ext }}(6)$. For air is also absolute pressure $p_{a}$ considered (7). The $h$ value depends on the geometry.

$$
\begin{gathered}
-\boldsymbol{n} *(-\boldsymbol{k} \boldsymbol{\nabla T})=\boldsymbol{h} *\left(\boldsymbol{T}_{\text {ext }}-\boldsymbol{T}\right) \\
\boldsymbol{h}=\boldsymbol{h}_{\text {water }}\left(\boldsymbol{L}, \boldsymbol{T}_{\text {ext }}\right) \\
\boldsymbol{h}=\boldsymbol{h}_{\text {air }}\left(\boldsymbol{L}, \boldsymbol{p}_{\boldsymbol{a}}, \boldsymbol{T}_{\text {ext }}\right)
\end{gathered}
$$

where:

$h-$ heat transfer coefficient, $\mathrm{W} /\left(\mathrm{m}^{2} \mathrm{~K}\right)$.

Fig. 1 shows the simulated setup as a three dimensional model. The aim of this simulation was to estimate how much will the total heat flux increase, when this model will be immersed into water instead of air. For the chosen point at the connection between the thermal grease and the heat sink there are two plots shown in fig. 2. Both present the simulated total heat flux in the time function - for air and water. Just after the junction is powered, it starts to dissipate heat so that the heat flux rapidly increases. After a few seconds the dissipated power needs to be reduced to avoid exceeding the $150^{\circ} \mathrm{C}$ junction temperature limit. Looking at the plots for air, the heat flux lowers very much, hence we conclude that the dissipated power at the transistor is also reduced very much. On the other hand with water cooling the heat flux remains stable at much higher level, thus the dissipated power is much higher in this case. Interpolating these two cases, it is expected that introducing water vapour into air will increase the possible heat flux without overheating the junction. In other words, it will enable higher power dissipation from the transistor with the same heat sink.

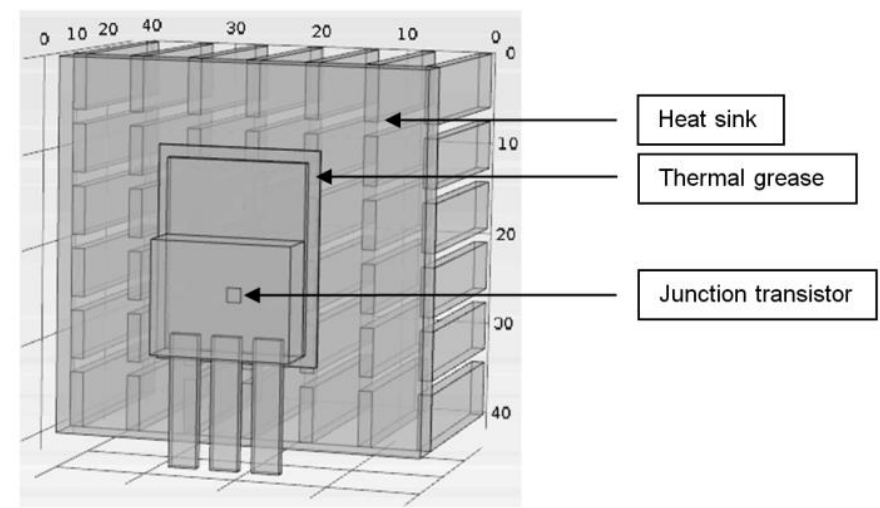

Fig. 1. The model set up including the heat sink, the transistor with the junction and the thermal grease 


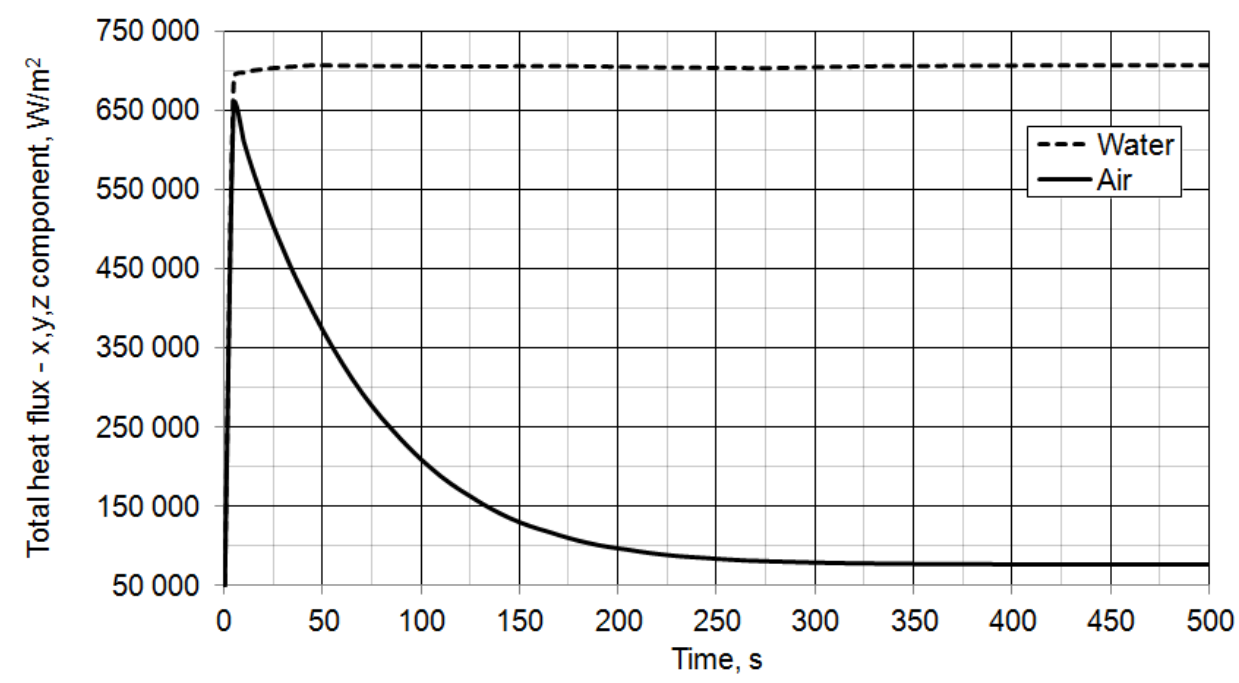

Fig. 2. The total heat flux in the time function for selected point of the structure

\section{Measurements}

To verify the stated hypothesis, the authors elaborated the test rig shown in fig. 3 . The chamber isolates the heat sink from the influence of surrounding disturbances that could affect the cooling conditions. The water vapour is produced by an ultrasonic generator, which consists of piezo-ceramic membrane located in a metal cabinet. This type of generator was chosen due to lower power consumption and quieter operation comparing to steam and evaporative generators. Thermal camera and cold background are for the purpose of convection flow visualization.

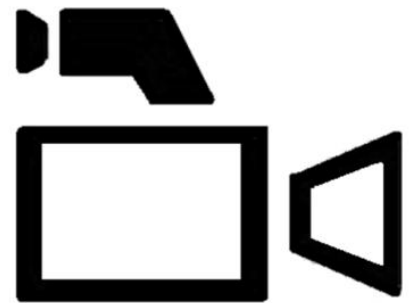

Infrared camera

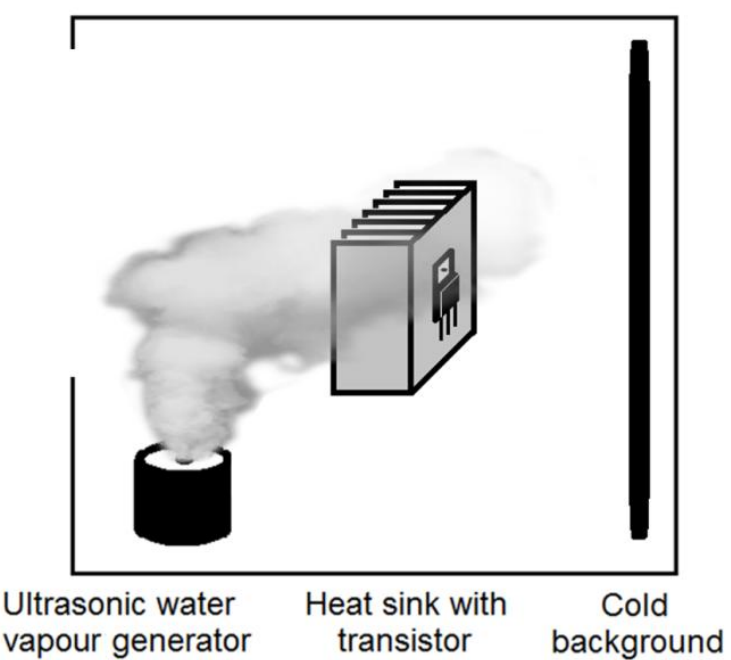

Fig. 3. The measurement test rig

The transistor is driven by a special circuit [6] that forces a constant current of $2 \mathrm{~A}$ trough the collector-emitter junction. Simultaneously the voltage drop over the base-emitter junction is measured by the controller. There is a known dependency of this voltage on the junction temperature $\left(-2.2 \mathrm{mV} /{ }^{\circ} \mathrm{C}\right)$. If it drops under a certain level the controller knows that the junction temperature exceeded the limit so it reduces the voltage drop on the collector-emitter junction (keeping the same current), what results in decreased power dissipation at the transistor.

As the controller acts to keep a constant voltage drop on base-emitter junction, it also keeps the constant junction temperature (set to $150^{\circ} \mathrm{C}$ ) regulating the dissipated power. This power is measured in real-time with a wattmeter by multiplying the constant current of collector-emitter junction and regulated voltage drop over this junction. In steady-state, it is the highest power that can be safely dissipated with particular heat-sink and thermal paste. However just after the power is turned on and the heat-sink is cold, the power readout is initially much higher and reduces as the heat-sink warms up to reach the steady-state. This behaviour is visible in fig. 4 . 


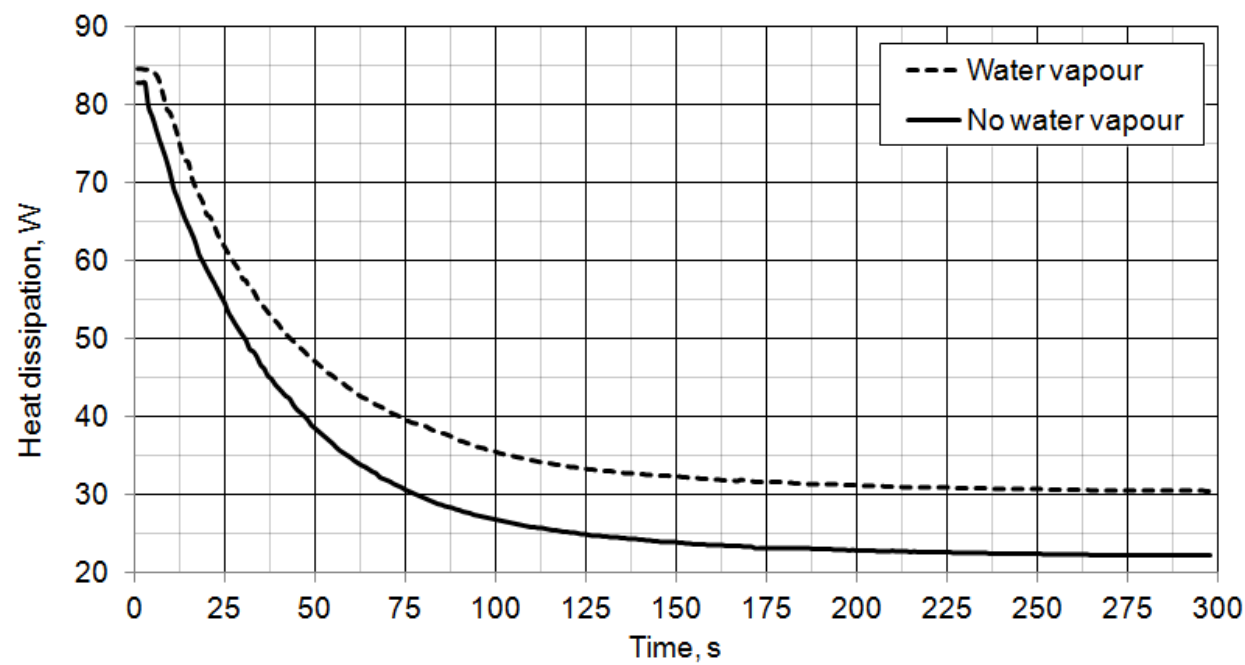

Fig. 4. The characteristic of power dissipation in the time function

As the heat sink temperature increases, it causes the surrounding air to warm up and move upwards due to convection. The authors measured the temperature of this convective flow (using Bestone BE856 data logger) for two cases - air and water vapour, as shown in fig. 5 . The difference is clearly visible - water vapour reaches about $10^{\circ} \mathrm{C}$ higher temperatures. This is because it takes more heat from the heat sink and hence cools it better allowing for higher power dissipation in the transistor.

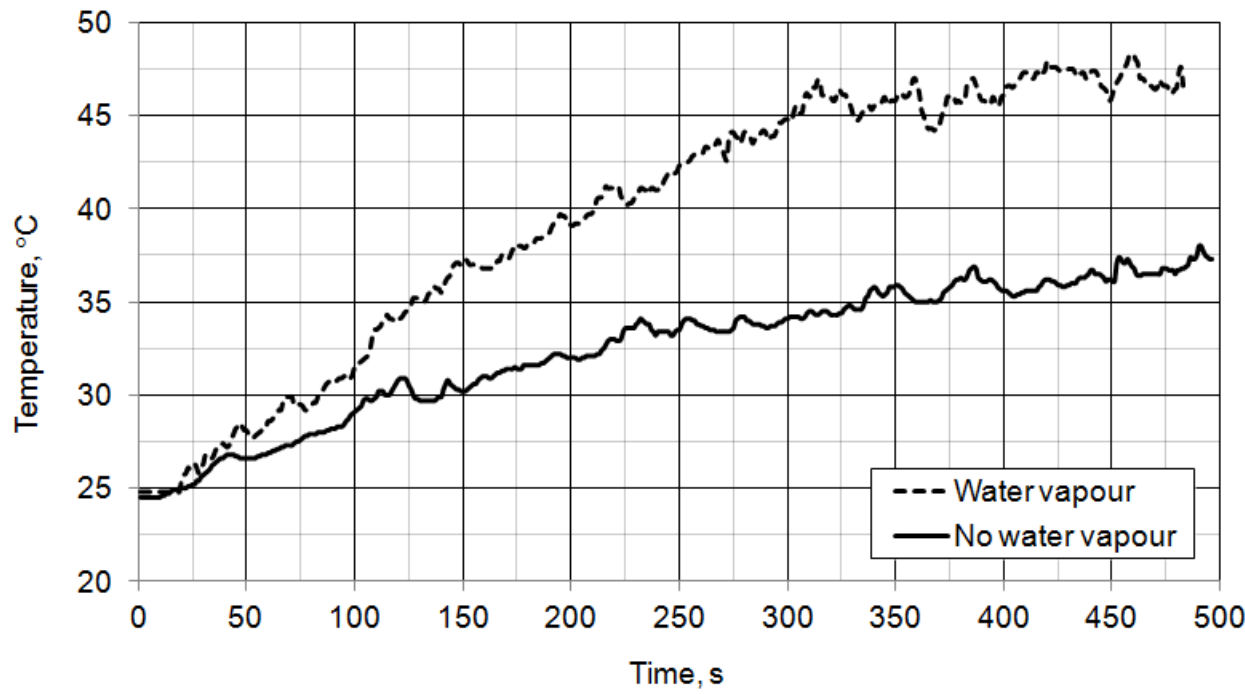

Fig. 5. Temperature of the convective flow over the heat sink in the time function

In parallel with temperature measurement of the convective flow also its humidity was measured with data logger Flus ET-175, as shown in fig. 6. In both cases the samples were recorded once per second. The measurements started after filling the bottom of the chamber with the water vapour. In the beginning the heat sink temperature was equal to the ambient. As it became hotter the convective flow appeared and in case of air its humidity started to gradually decrease. Opposite behaviour was observed for water vapour - with time recorded relative humidity increased up to $100 \%$. Having such humid convective flow the authors could even see it over the heat sink, as presented in the next paragraph. 


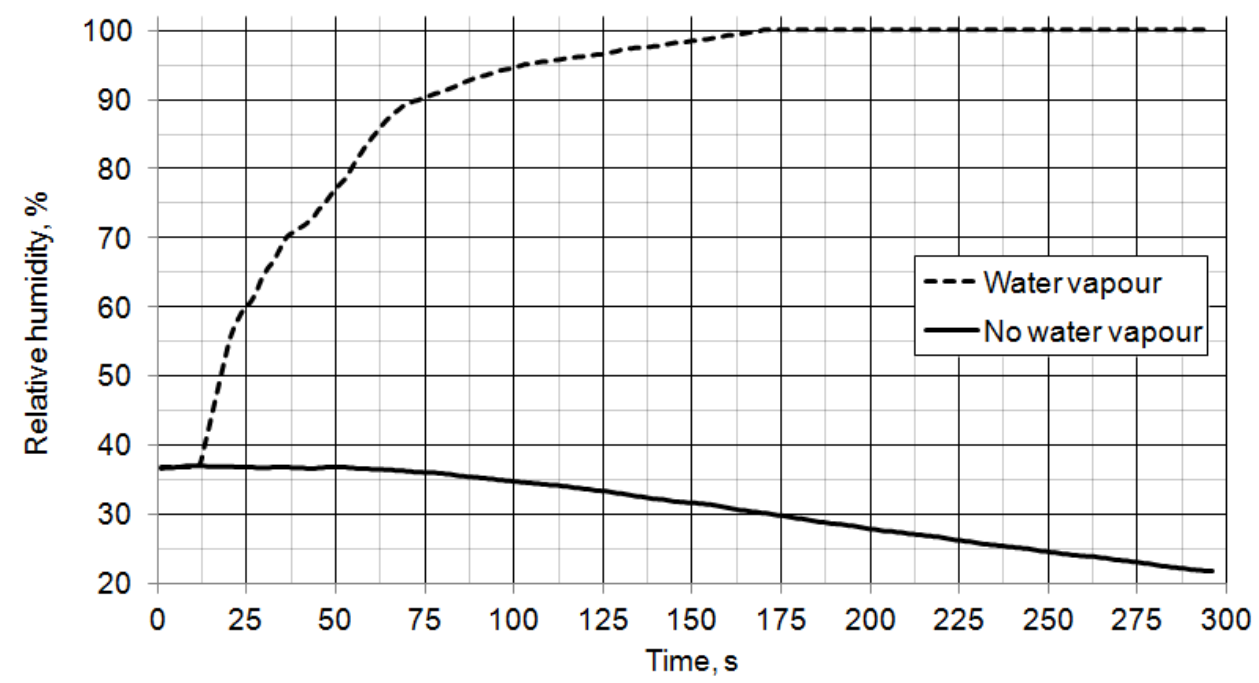

Fig. 6. Relative humidity of the convective flow over the heat sink in the time function

\section{Visualization of convection flow}

The convection flow of the water vapour over the heat sink was recorded in four different spectral ranges visible light, near infrared, mid-wavelength infrared and long-wavelength infrared, as shown in fig. 7 . To enhance the visibility of streams each image was processed with local equalization algorithm with the block of $25 \times 25$ pixels. It caused both the streams and the heat sink to have high contrast in a single photo.

Both visible and near infrared image was acquired with Basler 640 camera, but near infrared with IR720 filter. For MWIR range Cedip Titanium camera was used, and Jenoptik VarioCam for LWIR. In case of thermal cameras there was a cold background used to provide thermal contrast - temperature difference between the water vapour streams and the background was about $40^{\circ} \mathrm{C}$. If there was no contrast, it wouldn't be possible to see the streams. As far as the visible and near infrared spectral range is concerned, the streams are visible because of condensation which occurs when the water vapour floats over the heat sink.

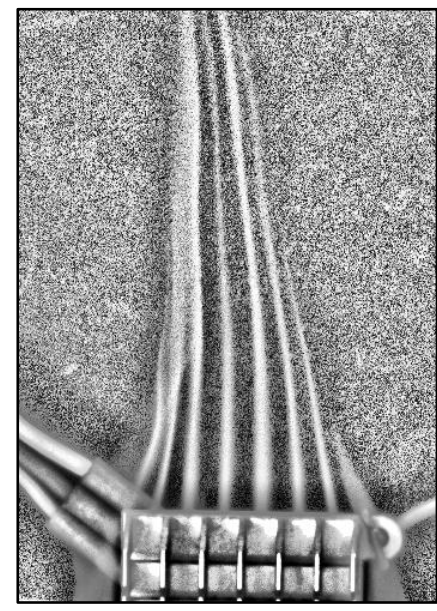

a) visible light

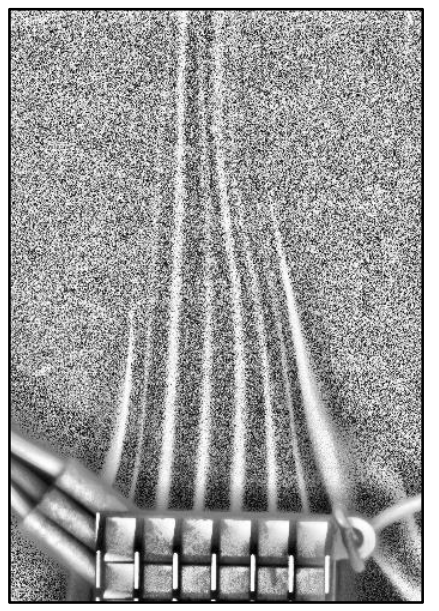

b) near infrared

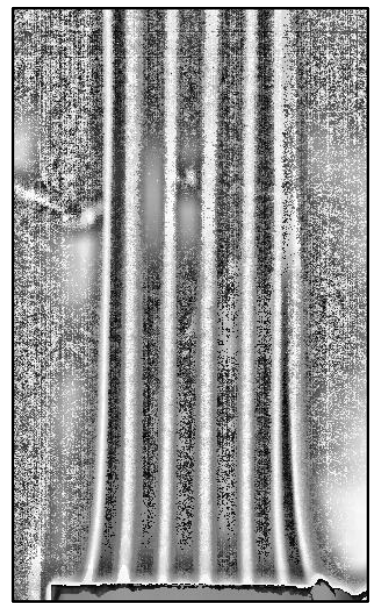

c) mid-wave infrared

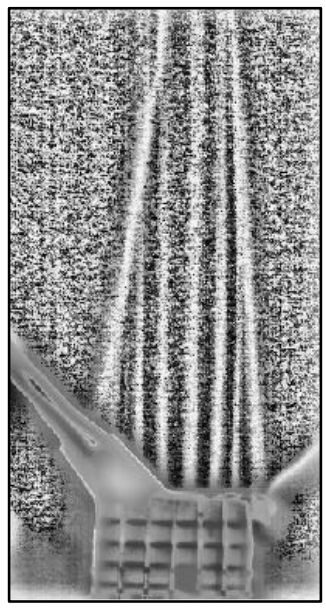

d) long-wave infrared

Fig. 7. The visualisations showing the water vapour flow in different spectral ranges (all images processed with local equalization algorithm)

\section{Analysis}

The first experiment was performed with traditional cooling without introducing the water vapour. The transistor junction temperature was kept at the level of $150^{\circ} \mathrm{C}$, which is maximum for this element. This condition was fulfilled by real-time monitoring this temperature and adjusting the dissipated power. In the beginning after the mains was switched 


\subsection{1/qirt.2016.061}

on, the dissipated power could be relatively high for a short period before the junction reached 150 ' $\mathrm{C}$. When it happened, the controller started to gradually reduced the dissipated power to avoid exceeding this temperature. It is clearly visible in fig. 4 (solid line). After reaching steady state the dissipated power was at the level of $22 \mathrm{~W}$.

The same experiment was repeated, but this time water vapour was introduced into the chamber. In the steady state the dissipated power was about $9 \mathrm{~W}$ higher than in case of traditional cooling without water vapour (fig. 4 - dashed line). In both cases the transistor junction had the same temperature equal to 150 ' $\mathrm{C}$. In this case introducing the water vapour to the chamber enabled about 35\% higher power dissipation in the transistor without increasing its junction temperature. This is because introducing the water vapour improves the cooling conditions causing the junction temperature to decrease, and it becomes possible to increase the dissipated power by about $35 \%$ to have the temperature again equal $150^{\circ} \mathrm{C}$.

During water vapour flow through the heat sink a lifting drift is formed and the internal energy is changed at the same time - the heat is transported from the element having higher temperature than the water vapour [4]. The reason is a pressure value, which is lower for the water vapour, than for the air [5]. The relationship is: the higher value of the pressure, the more densely packed molecules are. Therefore less space for other molecules is. The presence of the water vapour in the air influence on the higher amount of the absorbed energy, which leads to increased heat dissipation. On the basis of fig. 5 it can see that temperature of the air streams floating over the hat sink has higher value for the humid air. Undoubtedly the temperature mainly influence on the amount of the water molecules in the air. The rule is: the higher temperature of the air, the more water vapour in the air. After measurements of the air streams floating over the hat sink it was found that increase of the temperature causes increase amount of the water molecules in the air, consequently air humidity rises (fig. 6).

The obtained results also depends on the specific heat value. The specific heat is the amount of the energy $Q$ needed to increase temperature $T$ of the area in a unit mass $m$ by one unit and it is expressed:

$$
c(T)=\frac{1}{m}\left(\frac{\partial Q}{\partial T}\right)
$$

In the case of the real gases the specific heat value $C_{P}$ depends on the temperature and its value increases with the temperature. Therefore, it can't be used constant value of the specific heat for calculations. It should be used average value of the specific heat, i.e. the heat of the transformation from the temperature $T_{1}$ to the temperature $T_{2}$.

$$
\left.C_{p}\right|_{T_{1}} ^{T_{2}}=\frac{\left.C_{p}\right|_{0} ^{T_{2}} T_{2}-\left.C_{p}\right|_{0} ^{T_{1}} T_{1}}{T_{2}-T_{1}}
$$

When take $C_{P}$ of the air equal to $1005 \mathrm{~J} /\left(\mathrm{kg}^{*} \mathrm{~K}\right)$ and $C_{P}$ of the water vapour equal to $1840 \mathrm{~J} /\left(\mathrm{kg}^{*} \mathrm{~K}\right)$, then from the formula 8 and 9 it follows that the water vapour is able to absorb more energy that the air.

\section{Conclusions}

The principle of the proposed method is to improve the natural convection cooling conditions by introducing the water vapour to the surrounding of the heat sink. On the measurements can be deducted that introducing water vapour decreases the value of $R_{\text {thra. }}$. Hence:

- higher power may be dissipated in the transistor junction (about 35\% according to the measurements) without increasing its temperature and size of the heat sink, or

- temperature of the junction may be lowered with the same power dissipated, hence the MTBF parameter (mean time between failures) may be significantly increased, or

- size of the heat sink may be reduced while keeping the same dissipated power and junction temperature.

In addition, heat transfer of this process is realized mainly by natural convection and the results of air flow visualisation with multispectral system were presented. Air flow channels between the ribs of the heat sink are clearly visible in all spectral bands.

\section{REFERENCES}

[1] Xiang L., Wang Y., Wang R., Sun X., Wang R., "Influence of Air Humidity on thermal performance of radiator in armored vehicle". Applied Mechanics and Materials, vol. 71-78, pp. 2309-2314, July 2011.

[2] Incropera F., "Convection heat transfer in electronic equipment cooling". Journal of heat transfer, vol. 110, pp. 1097-1111, 1988.

[3] Awbi H., "Calculation of convective heat transfer coefficient of room surfaces for natural convection", Energy and buildings, vol. 28, pp. 219-227, 1998.

[4] Kitagawa K., Komoda N., Hayano H., Tanabe S., "Effect of humidity and small air movement on thermal comfort under a radiant cooling ceiling by subjective experiments", Energy and buildings, vol. 30, pp. 185-193, 1999.

[5] Lasance C. J. M., "The thermal conductivity of moist air". Test \& measurement, $1^{\text {st }}$ November 2003. [access on 02.04.2016]. Available online: http://www.electronics-cooling.com/2003/11/the-thermal-conductivity-of-moist-air/

[6] Piotr Górecki, Zbigniew Orłowski, Simple heat sink meter (in Polish: Prosty miernik radiatorów), Elektronika dla Wszystkich, AVT Publishing House, 2000. 